\title{
Collisional Simulations of Isolated Lindblad Resonances
}

\author{
J. HÄNNINEN and \\ H. SALO \\ Department of Astronomy, University of Oulu, SF-90570 Oulu, Finland \\ and \\ J. LUKKARI \\ Computer Services Centre, University of Oulu, SF-90570 Oulu, Finland
}

\begin{abstract}
.
The influence of the perturbing satellite on the planetary ring at isolated Lindblad resonances is studied with numerical computer simulations, combining the Aarseth's force polynomial method for orbit integrations with the calculation of particle-particle impacts. Observed angular momentum exchange between the satellite and the dissipative, non-selfgravitating ring agrees with the Goldreich-Tremaine formula for gravitating ring within $20 \%$, verifying that the exerted torque is not sensitive to the details of the dominant physical processes. The theoretically predicted angular momentum luminosity reversal was also observed.
\end{abstract}

\section{Introduction}

Dominant feature of all planetary ring systems is their wealth of radial structure. It seems evident that most of the regular density variations are generated by external satellites. Also, the location of the inner edge of Cassini Division suggests a close connetion with Mimas 2:1 Lindblad resonance. Satellites have also been proposed to explain the stability of narrow rings against viscous spreading, as well as many azimuthally asymmetric features.

The strongest interaction between ring and satellites takes place at narrow resonance zones. The behaviour of collisional systems in these regions has been extensively studied by Borderies, Goldreich, and Tremaine in a series of papers (Borderies, Goldreich, and Tremaine, 1982; 1983; 1985; 1986; 1989). For example, they have derived expressions for the angular momentum exhange between the ring and the satellite, caused by the torque exerted on the satellite-generated spiral density wave propagating outward from the resonance due to the ring self-gravity. However, self-gravity is not essential in this process (Goldreich and Tremaine, 1982; MeyerVernet and Sicardy, 1986): angular momentum exhange should occur if there is any process capable of creating a non-symmetric density response of the ring with respect to the rotating satellite potential. In the planetary rings this process can be either the self-gravity of the ring particles or just the interparticle collisions.

In this paper the dynamics of the ring-satellite resonances is studied in terms of numerical simulations. Our integration algorithm is based on Aarseth's N-body integrator (Aarseth, 1972; 1985), which uses fourth-order force polynomial and individual time-step schema in the integration of particle orbits. This code is very suitable for calculation of arbitrary satellite perturbations, also in the case of strong perturbations. Some modifications, taking advantage of the ring-shaped geometry have been made, and most importantly, we have included the calculation of particleparticle impacts. In principle, this can be done with very little extra CPU-time consumption, since in the construction of force polynomials mutual particle distances are calculated also in the original code.

S. Ferraz-Mello (ed.), Chaos, Resonance and Collective Dynamical Phenomena in the Solar System, 97-102. (C) 1992 IAU. Printed in the Netherlands. 


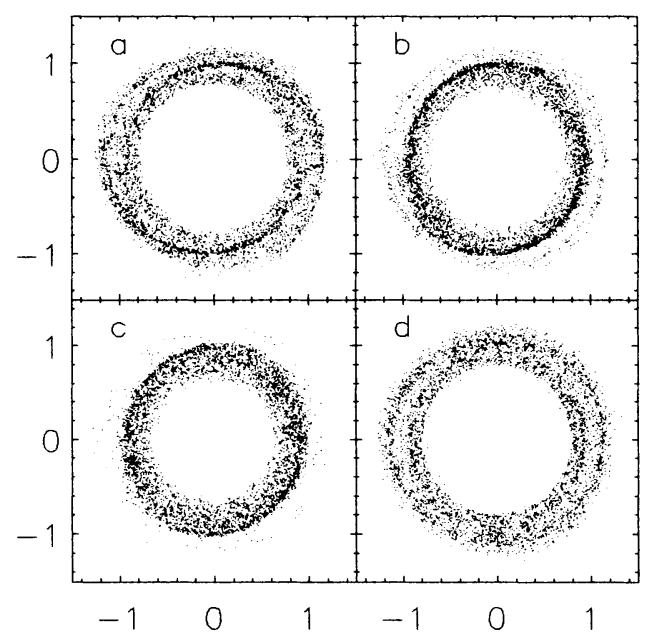

Fig. 1. The time evolution of the collisional ring at $2: 1$ resonance with external satellite is shown in frames a, b, and c with $t=5-25,95-115,295-315$ ring periods, respectively. The satellite $\left(M_{s}=2.5 \cdot 10^{-3} M_{p}\right)$ is located at three o'clock. The frame d shows collisionless ring as a comparison.

\section{Angular Momentum Transfer}

Besides affecting particle eccentricities, m:m-1 Lindblad resonance perturbations lead to the formation of azimuthal density variations with $m$-fold symmetry, stationary in the frame co-rotating with the satellite (Fig. 1). In the collisionless case the density wave appears symmetric with respect to the satellite, and consists of $m$ equally spaced loops (Fig. 1d displays the collisionless $m=2$ case), following from the $180^{\circ}$ phase shift in the alignment of particle orbits at various sides of the exact resonance radius. In the collisional case the discontinuity in the alignment is removed, leading to the gradual formation of trailing spiral density wave appearing stationary in the coordinate system corotating with the satellite. This fact makes it possible to improve the resolution of the simulation data by averaging over long time spans when studying the physical properties of the ring.

In addition to the stationary density wave there is also noticeable time evolution in the radial distribution of particles. The particles orbiting initially outside the resonance distance fall inward due to the total effect of satellite perturbations and collisions. For example, in Fig. 1a-c, practically all particles are eventually cleared out from the outer parts of the ring, and a sharp outer edge is formed at the 2:1 resonance radius. On the macroscopic level this infall can be intepreted to be due to the torque excerted by the satellite on the non-axisymmetric density response of the ring, transferring angular momentum of the ring particles to the satellite (in the case of ILR). In terms of individual particle orbits, conservation of Jacobi constant requires that the excitation of eccentrities is connected with a slight decrease in 


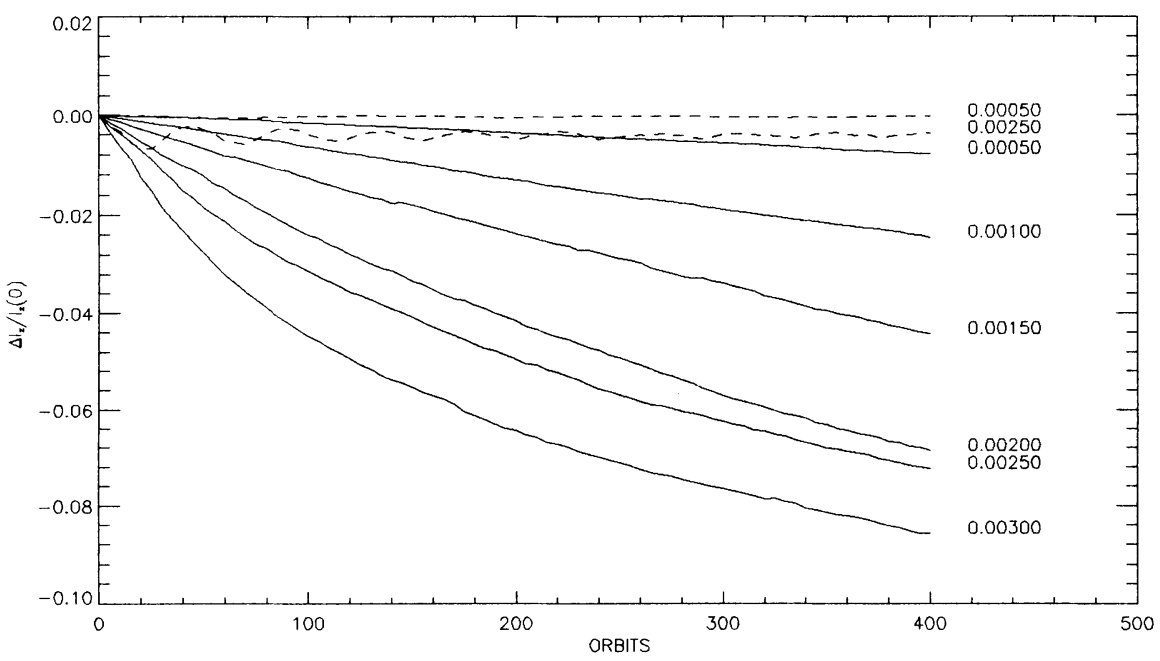

Fig. 2. The relative angular momentum change is plotted for 6 different satellite masses. The two dashed curves correspond to collisionless simulation. The ring of 800 identical massless particles with radius $5 \cdot 10^{-3}$ has an initial width $W=0.4$.

the semi-major axis of the particle. If at least part of the eccentricity is damped by impacts, cumulative change of $a$ is obtained (Dermott, 1984).

The angular momentum exchange between the satellite and the ring in an inner 2:1 Lindblad resonance has been systematically examined in a series of numerical simulations where the initial optical depth of the ring is $\tau=0.025$. At the beginning of the simulations the collisionless and the collisional cases yield the same decrease of angular momentum. This transient stage of evolution follows from the "creation" of the satellite at time $t=0$ : the nonstationary evolution of the ring allows the satellite to exert net torque on the ring (Meyer-Vernet and Sicardy, 1986). However, after the short nonstationary phase the net torque vanishes in the collisionless simulations, since the formed density feature is symmetric with respect to satellite (see Fig. 1d). On the other hand, in the case of colliding particles the ring-response is non-symmetric with respect to satellite, leading to continuous removal of angular momentum from the ring, in a rate (proportional to the square of the mass of satellite) which at the beginning of the simulation is approximately constant but later decreases. This latter reduction results from the reduced surface density in the resonance area during the course of the simulation (see Fig. 1), as the simulation system as a whole moves inward. As can be expected, the nonlinearity is largest in the case of the most massive satellite. In the linear regime the simulated torque is within $20 \%$ of the standard Goldreich-Tremaine formula.

The oscillations seen in the collisionless case are most probably due to the small number of simulation particles. The particles possess several different perturbation periods diversing from the angular speed of the resonance pattern, and depending on their net orbital stage when approaching and leaving the pattern the instantenous 


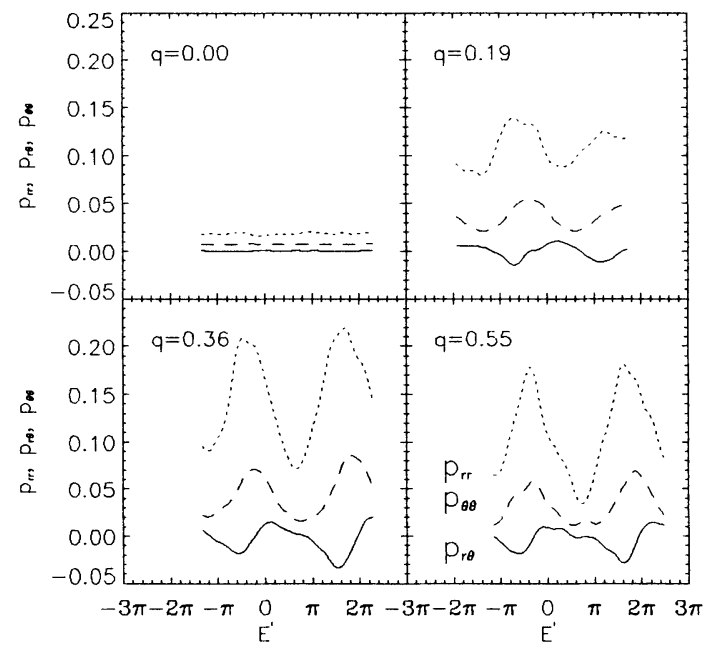

Fig. 3. The viscous stress tensor components are plotted for 4 different values of nonlinearity parameter $q$. The components are calculated from the collisional 2:1 inner Lindblad resonance simulation, except for the value $q=0.0$ which has been taken from the perturbationless simulation. They are plotted as a function of $E^{\prime}$, distance from density maximum.

direction of the exerted torque can vary. The amplitude of the oscillation depends on the mass of satellite but it vanishes if the number of particles is increased.

\section{Perturbed Streamlines}

The ring particle orbits near resonance can be conveniently described by streamline formalism (Borderies, Goldreich, and Tremaine, 1982; 1983; 1985; 1986). In a cylindrical coordinate system $(r, \phi)$ the shape of a streamline can be written as $r=a[1-e(a) \cos (m(\phi+\Delta(a)))]$, where $a$ is the semimajor axis, $e$ is the eccentricity $(e \ll 1), \Delta$ is a phase angle, and $m$ is azimuthal wavenumber $(m>0)$ characterizing the ring-satellite resonance. The coordinate system corotates with the satellite. We use the notation of Borderies, Goldreich, and Tremaine throughout the text in order to be able to compare simulations with theoretical results: the symbol $E^{\prime}$ is used for the distance from density maximum along each streamline, and $q$ stands for the nonlinearity parameter. In a nonperturbed case $q=0$. If $q \geq 1$, the streamlines intersect which is not possible for a dissipative system with even modest optical depths.

The behaviour of the streamlines was studied in detail for 2:1 ILR simulation with $M_{s}=2.5 \cdot 10^{-3} M_{p}$. When using 800 ring particles with a radius $\sigma=0.005$, the optical depth $\tau=0.025$ was obtained. This number of particles is still too small to give good resolution. However, by collecting data over several orbital periods, enough resolution was obtained to enable the accurate fitting of streamlines to the particle distribution as well as calculation of velocity ellipsoids and densities along 


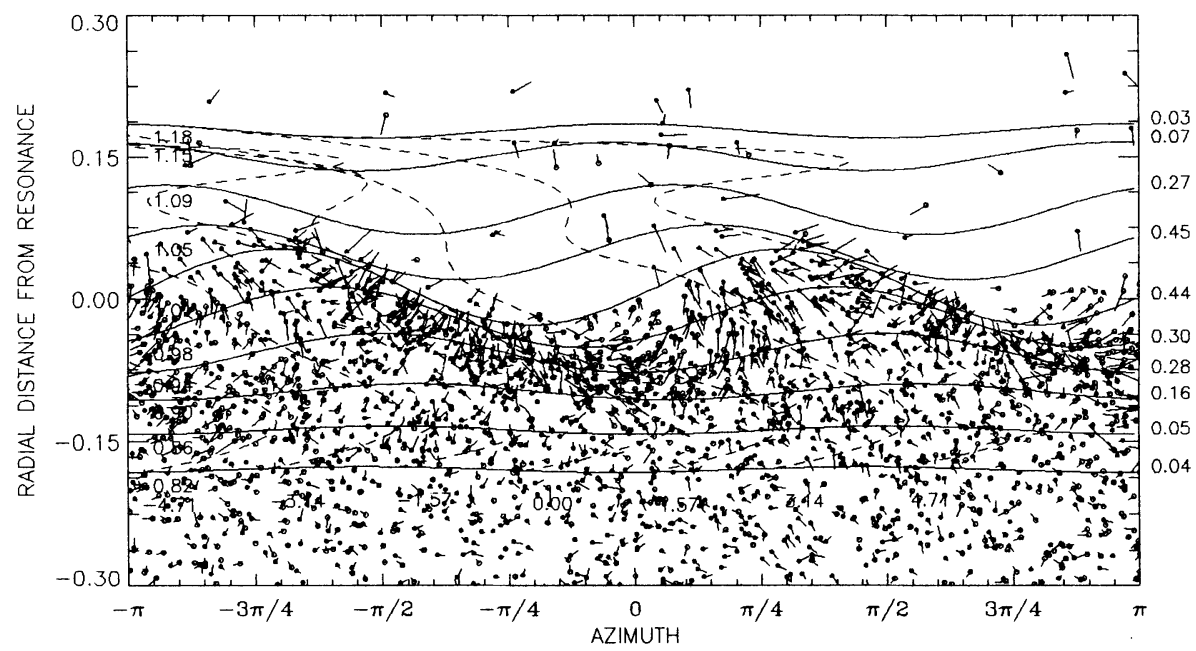

Fig. 4. The velocity deviation from the Keplerian velocity is shown with a tail attached to the ring particle. Also the least square fits of the streamlines are shown with appropriate values for nonlinearity parameter $q$ (right edge) and semimajor axis (left edge). Also some constant value curves for $E^{\prime}$ are plotted with dashed lines. The ring is in inner 2:1 Linblad resonance with the satellite having mass of $M_{s}=2.5 \cdot 10^{-3} M_{p}$.

streamlines. From these fits also non-linearity factors can be derived.

In the Fig. 3 the azimuthal variation of the components of the viscous stress tensor $\mathbf{p}$ (product of density and velocity dispersion tensor) along a streamline is shown for four different values of $q$, plotted as a function of $E^{\prime}$. The viscous angular momentum flux $F$ is the rate at which the particle-particle impacts transport angular momentum across a unit length of streamline with semimajor axis $a$ at longitude $\phi$. It is calculated directly from the non-diagonal component of the viscous stress tensor (Borderies, Goldreich, and Tremaine, 1982), $F(a, \phi)=a p_{r \theta}(a, \phi)$.

At $q=0$ the component $p_{r \theta}$ has small positive values around the streamline without any significant azimuthal variation. There is only normal outward directed collision induced angular momentum transport caused by differential rotation. When satellite perturbations have caused the nonlinearity to increase into $q=0.19$ the viscous stress components are clearly increased, and also show strong azimuthal variations. Inspection of the non-diagonal component $p_{r \theta}$ shows the reversal of the angular momentum flux at some longitude intervals (compared to Figs. 3-5 in BGT, 1983). At larger values of $q$ the azimuthal fluctuations are increased, and $p_{r \theta}$ has larger negative values. There are no large differences in the viscous stress tensor, when the nonlinearity factor grows from $q=0.36$ to $q=0.55$ even if the perturbation is stronger in the latter case. When the $q$ value is larger, there is also larger velocity dispersion, but the density is also smaller because of the larger 
perturbations. These changes compensate each other, and there is no significant differences in viscous stress tensor components. When the along streamline integrated angular momentum flux, the angular momentum luminosity is negative, the net angular momentum flow is directed inward, and a gap or sharp edge is formed in the ring. This phenomenon has been verified by our simulations.

The wavy streamlines in the perturbed ring are seen as satellite generated peculiar velocites of individual particles on the microscopic level. In Figure 4 the least square fits to the streamlines are shown with $q$ and some constant values of $E^{\prime}$. The individual particles are plotted with a tail showing the velocity deviation from the Keplerian velocity. Particles with positive tangential velocity difference are plotted as open circles. The azimuth of maximum inward directed angular momentum flux (at $E^{\prime} \simeq-\pi / 2$, see Fig. 3 ) is seen to correspond to the inner edges of density waves in which there are particles with strong inward directed peculiar velocities.

\section{References}

S. J. Aarseth, Gravitational N-Body Problem, 373, (1972).

S. J. Aarseth, Multiple Time Scales, 377, (1985).

N. Borderies, P. Goldreich and S. Tremaine, Nature, 299, 209, (1982).

N. Borderies, P. Goldreich and S. Tremaine, Icarus, 55, 124, (1983).

N. Borderies, P. Goldreich and S. Tremaine, Icarus, 63, 406, (1985).

N. Borderies, P. Goldreich and S. Tremaine, Icarus, 68, 522, (1986).

N. Borderies, P. Goldreich and S. Tremaine, Icarus, 80, 344, (1989).

S. F. Dermott, Planetary Rings, 589, (1984).

P. Goldreich and S. Tremaine, Ann. Rev. Astron. Astrophys., 20, 249, (1982).

N. Meyer-Vernet and B. Sicardy, Icarus, 69, 157, (1986).

\section{Discussion}

P.Goldreich - I have only a comment. This is beautiful work, the first convincing particle simulation of the dynamics of sharp edges. 